\title{
Challenges in Aggression Assessment: The Gap between Self-report and Behavior, and a Call for New Valid Behavioral Paradigms
}

Jill Lobbestael *

Department of Clinical Psychological Science, Faculty of Psychology and Neuropsychology, Maastricht University, The Netherlands

\section{Reactive and Proactive Aggression}

Aggression is defined as any behavior directed towards another individual that is carried out with the intention to cause harm. The perpetrator must also believe that his/her behavior will harm the target and the target must be motivated to avoid this behavior [1,2]. Aggression is not a unitary concept. Subdivisions can for example be made based on the type of expression (e.g. verbal or physical) or on contextual aspects (e.g. relational). A motivation-based distinction can be particularly appealing because it refers to the origin of aggressive behavior, and therefore designates avenues for therapeutic interventions.

One bimodal motivational differentiation is that of reactive versus proactive aggression. Reactive aggression has been characterized as 'hot-blooded' and refers to impulsive, fear-induced, anger-driven defensive responses to a real or perceived provocation. It is opposed by proactive aggression, the 'cold-blooded' form of aggression installed to achieve a certain goal, like power or money. Proactive aggression is instrumental, organized and premeditated [1,2]. Importantly, a person can display both reactive and proactive aggression, even in one and the same aggressive incident (e.g. a planned robbery where a perpetrator impulsively hits one of the hostages because he feels insulted by that person). Consequently, both aggression types should be considered as two separate dimensions, instead of opposing concepts [3]. Both aggression types have received increased empirical attention during the last decades, and research has linked both types to distinct developmental precursors, behavioral and psychopathological concepts [4].

\section{Assessment of Reactive and Proactive Aggression}

The majority of studies operationalized reactive and proactive aggression using self-report questionnaires. Examples of questionnaires among adult populations are the Reactive Proactive Questionnaire [5], and the Impulsive/Premeditated Aggression Scale [6]. Although questionnaires like these have the clear advantage of being short and quickly to apply and posit few ethical challenges, they can be considered suboptimal. The most prominent reasons for this are that aggression is highly socially unacceptable, and therefore particularly likely to be underreported on because of social desirability. Thus, people might be reluctant to admit engaging in aggressive behavior in order to keep a positive social status. Additionally, self-report of aggression could be unreliable due to lack of insight.

In response to these drawbacks of self-report measures, several behavioral measures of aggression have been developed. Among the most often used are the Taylor Aggression Paradigm [7], the Point Subtraction Aggression Paradigm [8] and the hot-sauce paradigm [9]. The TAP is a competitive reaction game against a virtual opponent where participants have to try to be the first to press a button after a red square appears on the computer screen. In case they supposedly win a trial, they can administer an aversive tone through headphones to their opponent. The louder and longer this tone, the more aggression. In the PSAP, participants can earn points with pressing a button 100 times, but they can also steel points from their fictive opponent with less effort (i.e. pressing another button 10 times). The more often they opt for the latter tactic, the more aggressive they are. Finally, in the hot-sauce paradigm, participants are supposedly making a sauce for another participant in a tasting experiment. The more extremely hot sauce they administer to their opponent (while knowing the opponent dislikes hot food), the more aggression. While these tasks were not specifically developed for assessing reactive and proactive aggression, all three of them can be easily modulated to include a provoked variant measuring reactive aggression (i.e. first receiving highly aversive tones from their opponent in the TAP or having points stolen from them by their opponent in the PSAP, and having the prepare a sauce after their opponent gave them negative feedback on a previous, supposedly unrelated essay writing task in the hot-sauce paradigm). While merely leaving out these additional provocation aspects provides an assessment of unprovoked aggression, importantly, this is not sufficient to adequately assess proactive aggression. Specifically, proactive aggression requires the use of aggression to obtain a specific goal, so behavioral assessment tasks should be modified in such a way that participants are motivated to use aggression instrumentally e.g. in order to obtain money or power.

\section{What People Say is Not what they Do: Lack of Correlations}

One striking and recurring finding is that self-reported levels of aggression hardly correlate with behaviorally assessed aggression. In case of reactive and proactive aggression, we are only aware of studies simultaneously using both the RPQ and the TAP in adult samples $[2,10]$. There are several possible reasons that could explain this selfreport/behavior gap. First, both assess different time frames; the RPQ assesses trait aggression, while the TAP is a momentary assessment, and thus a state measure. Second, the TAP specifically taps into physical aggression (i.e. administering aversive tones), while the RPQ also refers to vandalism or verbal aggression. Giancola and Parrott [11] refer to a similar argument in the general aggression literature, namely that most self-report measures on aggression also include items on anger or hostility, making them less 'clean `aggression instruments. Third, filling out the RPQ might be conceived by participants as more anonymous, in the sense that the experimenter does not directly witness a participant's aggression as can be the case in behavioral assessment paradigms. Fourth, the RPQ is very transparent about measuring aggression, while this is not the case in behavioral assessment paradigms. Instead, rather complex cover stories are used in behavioral aggression paradigms.

*Corresponding author: Jill Lobbestael, Department of Clinical Psychological Science, Faculty of Psychology and Neuropsychology, Maastricht University, PO Box 616, 6200 MD Maastricht, The Netherlands, E-mail: Jill.Lobbestael@maastrichtuniversity.nl

Received December 12, 2015; Accepted December20, 2015; Published December 28,2015

Citation: Lobbestael J (2015) Challenges in Aggression Assessment: The Gap between Self-report and Behavior, and a Call for New Valid Behavioral Paradigms. J Socialomics 5: 141. doi:10.4172/2167-0358.1000141

Copyright: ( 2015 Lobbestael J. This is an open-access article distributed under the terms of the Creative Commons Attribution License, which permits unrestricted use, distribution, and reproduction in any medium, provided the original author and source are credited. 
The self-report/behavioral gap in aggression assessment raises the question which of both assessment types most accurately reflects the true level of aggression. One the one hand, the higher levels of perceived anonymity in the self-report measures could render these measures as more accurate. One the other hand, there are two strong arguments that render the superiority of behaviorally measured aggression; (i) the fact that it is less clear that the socially unacceptable behavior of aggression is measured, and (ii) the fact that participants are actually exposed to aggression-triggering situations, instead of them being inquired about in hypothetical situations. Because of these reasons, the extra effort of behaviorally assessing aggression can be considered a worthwhile endeavor.

\section{A New Valid Alternative: Virtual Reality}

Although the three behavioral methods outlined above each overcome disadvantages criticized in several other aggression research methods, they still have two important disadvantages. First, none of them involve the direct physical attack of another individual. Second, they require complicated cover stories, and become completely invalid in case a participant sees through the cover story of the presence of an actual other opponent. In our experience, the latter happens in at about $10 \%$ of the cases, despite extended efforts (e.g. including a second experimenter supposedly supervising the second participant; taking pictures of the participant and sending it out to the other experimenter as well as showing the participant pictures of the socalled opponent). One particular promising candidate to validly assess behavioral aggression is virtual reality (VR). VR involves the creation of a 3-dimensional immersive environment in which individuals can freely move and interact. VR is fairly new applied in psychopathological research, and to our best knowledge, our group is the first to develop a VR aggression assessment paradigm.

There are several advantages of VR assessment of aggression. First, VR scenarios can be constructed in a way that renders the use of cover stories unnecessary. Second, because the victim of aggression in the VR paradigm is not an actual person, there is no harm doing involved, and there is no need to create a distance between the participant and the victim. This not only renders the VR task more realistic, but also makes it free of ethical constraints. Third, VR environments are both highly standardized, and highly modifiable, which allows assessing aggression e.g. in different environments and in response to different types of avatars (e.g. male/females, different ages and nationalities). Finally, participants become immerged or 'telepresent' in the virtual environment, and tend to forget about the actual world surrounding them. This imposes a feeling of privacy (in contrast to an unnatural laboratory environment) increasing the likelihood of participants presenting real-life behavior.

In our lab, we developed a head-mounted display VR pub environment where the participant observes two avatars (i.e. virtual persons) playing a game of darts. In the first part of the reactive condition, the participant has to put a bet on which player will win. The VR task is constructed in such a way that the player the participant did not bet on, cheats during the game. In the first part of the proactive condition, the participant merely observes two avatars playing the darts game. In the second part of the reactive game condition, the participant again meets the cheating avatar, who provokes the participant by laughing at him for betting on the wrong player. The participant has the choice to either not react, verbally react (aggressively), or hit the avatar. In the second part of the game in the proactive condition, the participant also again meets one of the avatars, and can opt to challenge the avatar to a darting game where he can win 3 euros; start a fistfight with the avatar to receive 6 euros; or hit the avatar with a bottle to receive 10 euros. The first findings are promising in the sense that scores of both reactive and proactive forms of aggressiveness assessed in the VR tasks correspond with self-reported levels of both aggression types, suggesting good convergent validity. Furthermore, internal consistencies of both aggression types assessed with VR were adequate.

\section{Avenues for Future Research}

At the moment, there are no studies incorporating both self-report questionnaires to assess reactive and proactive aggression together with multiple behavioral aggression paradigms. It is therefore unknown how the reactive and proactive subscales of self-report aggression measures correspond with behavioral tasks other than the TAP, nor do we know how different subscales of the behavioral tasks intercorrelate. Knowledge on this matter could provide researchers with empirical reasons which specific type of behavioral aggression task to choose.

We also call for further research to answer the question why there is a lack of converge between self-reported and behaviorally assessed aggression. One potential candidate contributing to this convergence is social desirability. We are only aware of one study in this area that revealed that individuals who reported more social desirability, reported less aggression [12]. To our knowledge, it is currently unknown how social desirability would impact behaviorally assessed aggression. Likewise, it would be interesting to empirically test the impact of anonymity on aggression assessment measures.

Finally, VR seems a promising behavioral assessment measure. More studies are needed in this area though, as is the exploration of VR applications for training health care professionals, or therapeutically application in the area of aggression.

\section{References}

1. Anderson CA, Bushman BJ (2002) Human aggression. Annual Review of Psychology 53: 27-51.

2. Bushman BJ, Anderson CA (2001) Is It Time to Pull the Plug on the Hostile Versus Instrumental Aggression Dichotomy? Psychological review 108: 274.

3. Poulin F, Boivin M (2000) Reactive and proactive aggression: evidence of a two- factor model. Psychological Assessment 12: 115-122.

4. Cima M, Raine A (2009) Distinct characteristics of psychopathy relate to different subtypes of aggression. Personality and Individual Differences 47: 835-840.

5. Raine A, Dodge K, Loeber R, Gatzke kopp L, Lynam D, et al. (2006). The reactiveproactive aggression questionnaire: differential correlates of reactive and proactive aggression in adolescent boys. Aggressive Behavior 32: 159-171.

6. Babcock JC, TharpALT, Sharp C, HeppnerW, Stanford MS, etal. (2014) Similarities and differences in impulsive/premeditated and reactive/proactive bimodal classifications of aggression. Aggression and Violent Behavior 19: 251-262.

7. Taylor S (1967) Aggressive behavior and physiological arousal as a function of provocation and the tendency to inhibit aggression. Journal of Personality 35: 297-310.

8. Cherek D (1981) Effects of smoking different doses of nicotine on human aggressive behavior. Psychopharmacology 75: 339-345.

9. Lieberman JD, Solomon S, Greenberg J, McGregor HA (1999) A hot new way to measure aggression: Hot sauce allocation. Aggressive Behavior 25: 331-348.

10. Lobbestael J, Baumeister R, Fiebig T, Eckel LA (2014) The role of grandiose and vulnerable narcissism in self-reported and laboratory aggression and hormonal reactivity. Personality and Individual Differences 69: 22-27.

11. Giancola PR, Parrott DJ (2008) Further evidence for the validity of the Taylor Aggression Paradigm. Aggressive Behavior 34: 214-229.

12. Vigil-Colet A, Ruiz-Pamies N, Anguiano-Carrasco C, Lorenzo-Sevo U (2012) The impact of social desirability on psychometric measures of aggression. Psicothema 24: 310-315 